\title{
A criterion for the positivity of the Liapunov characteristic exponent
}

\author{
ERIC CORNELIS AND MACIEJ WOJTKOWSKI
}

Department of Mathematics, Facultés Universitaires Notre Dame de la Paix, Rempart

de la Vierge, 8, B-5000 Namur, Belgium; Department of Mathematics, University of Arizona, Tucson, AZ 84721, USA and University of Warsaw, Poland

\section{(Received 14 October 1983)}

Abstract. We formulate sufficient conditions under which, for a finite subset of $\mathrm{SL}(2, \mathbb{R})$, the maximal Liapunov exponent is positive. These conditions are based on the notion of compatible hyperbolicity. We then give an analytical formulation of such a condition and we apply this criterion to prove mixing properties of a particular transformation of the two-dimensional torus.

\section{Introduction}

Let $X$ be a measure space with a probability measure $\mu$ and let $T: X \rightarrow X$ be a measure preserving transformation. Let $A: X \rightarrow \operatorname{SL}(2, \mathbb{R})$ be a measurable mapping. The maximal Liapunov characteristic exponent (m.L.c.e.) is by definition

$$
\gamma^{+}(x)=\lim _{n \rightarrow+\infty} \ln \left\|A\left(T^{n-1} x\right) \cdots A(x)\right\| .
$$

Oseledec's multiplicative ergodic theorem [2] asserts that $\gamma^{+}(x)$ exists almost everywhere (at least if $A(X)$ is bounded). The significance of the m.L.c.e. for the study of mixing properties of dynamical systems is now well established [3]. In this paper, we consider the case of a finite set $A(X)=\left\{A_{1}, \ldots, A_{n}\right\} \subset \operatorname{SL}(2, \mathbb{R})$ and formulate sufficient conditions under which the $m$.L.c.e. is positive. These conditions actually mean that, in some basis, all matrices from $A(X)$ have positive entries except for parabolic matrices which have only non-negative entries. We give an analytic formulation of such a condition. In particular, our criterion depends only very weakly on properties of $T: X \rightarrow X$. The criterion is an abstraction of methods used in proving positivity of the m.L.c.e. for some piecewise linear tranformations of the torus [4], [1], [5]. In $\S 3$, we give another application of the criterion in the same spirit.

Our discussion is centred on the concept of compatible hyperbolicity of a set of matrices $\left\{H_{1}, \ldots, H_{n}\right\} \subset s \ell(2, \mathbb{R})$ which we study thoroughly in $\S \S 1,2$.

In proposition 1 , we prove that the inverse of the exponental function in $\operatorname{SL}(2, \mathbb{R})$ is linear up to a multiplication by a scalar. This is a crucial analytic tool in our work.

\section{Compatible hyperbolicity}

Let us consider the group $\operatorname{SL}(2, \mathbb{R})$ of real $2 \times 2$ matrices with determinant equal to 1. $A \in \mathrm{SL}(2, \mathbb{R})$ is called a hyperbolic matrix if it has real eigenvalues different from 
1 and -1 , an elliptic matrix if it has a pair of complex conjugate eigenvalues different from 1 and -1 and, finally, it is called a parabolic matrix if it has eigenvalues equal to either 1 or -1 . Thus, we have that $A \in S L(2, \mathbb{R})$ is hyperbolic if $|\operatorname{tr} A|>2$, elliptic if $|\operatorname{tr} A|<2$ and parabolic if $|\operatorname{tr} A|=2$.

$\Delta \ell(2, \mathbb{R})$ denotes the Lie algebra of $\operatorname{SL}(2, \mathbb{R})$. It consists of real $2 \times 2$ matrices with zero trace. $H \in \Delta \ell(2, \mathbb{R})$ is called hyperbolic if $e^{t H} \in \operatorname{SL}(2, \mathbb{R})$ is hyperbolic for all real $t \neq 0$. Analogously, we define elliptic and parabolic elements of $s \ell(2, \mathbb{R})$.

The exponential function $\exp : \triangleleft \ell(2, \mathbb{R}) \rightarrow \operatorname{SL}(2, \mathbb{R})$ maps $\diamond \ell(2, \mathbb{R})$ onto $\{A \in \operatorname{SL}(2, \mathbb{R}) \mid \operatorname{tr} A>-2$ or $A=-I\}$. Moreover it is $1-1$ on the subset of hyperbolic matrices. The following proposition states that the inverse function is linear up to multiplication by a scalar.

Proposition 1. Let

and

$$
\left(\begin{array}{ll}
a & b \\
c & d
\end{array}\right)=A \in \operatorname{SL}(2, \mathbb{R}), \operatorname{tr} A>-2
$$

$$
\left(\begin{array}{cc}
\frac{(a-d)}{2} & b \\
c & \frac{(d-a)}{2}
\end{array}\right)=H \in \diamond \ell(2, \mathbb{R}) ;
$$

then there is $t>0$ such that $e^{t H}=A$.

Proof. By straightforward computation, we have that the quadratic form $Q(x, y)=$ $-c x^{2}+(a-d) x y+b y^{2}$ on $\mathbb{R}^{2}$ is invariant under the action of $A: \mathbb{R}^{2} \rightarrow \mathbb{R}^{2}$.

Consider some non-zero quadratic form $\phi(x, y)=e x^{2}+2 f x y+g y^{2}$.

We want to determine all one parameter subgroups of $\operatorname{SL}(2, \mathbb{R})$ that preserve this quadratic form. For this purpose, let

$$
\left(\begin{array}{rr}
p & q \\
r & -p
\end{array}\right)=K \in \delta \ell(2, \mathbb{R}),
$$

and let $K$ be the linear vector field in $\mathbb{R}^{2}$ defined by $K$. Taking the Lie derivative, we obtain

$$
0 \equiv L_{\kappa} \phi(x, y)=2(e p+f r) x^{2}+2(e q+g r) x y+2(f q-g p) y^{2}
$$

Hence, we must have

$$
\begin{aligned}
& e p+f r=0 \\
& e q+g r=0 \\
& f q-g p=0
\end{aligned}
$$

since $\phi$ is preserved. For $e^{2}+f^{2}+g^{2}>0$ (i.e. $\{e, f, g\} \neq\{0\}$ ), these equations have an unique solution up to a multiplicative constant:

$$
p=f, \quad q=g, \quad r=-e .
$$

On the other hand, each $A \in \operatorname{SL}(2, \mathbb{R})$ with $\operatorname{tr} A>-2$ can be included in an unique one parameter subgroup of $\operatorname{SL}(2, \mathbb{R})$, except for

$$
I=\left(\begin{array}{ll}
1 & 0 \\
0 & 1
\end{array}\right)
$$


Also, for each $A \in \operatorname{SL}(2, \mathbb{R})$, except for $I$ and $-I$, there is an invariant quadratic form (unique up to a constant). So we must have $e^{t H}=A$ for some $t \in \mathbb{B}$ and $H$ defined above.

We have yet to prove that we can choose $t>0$. For elliptic $A, t$ is determined modulo the period; so clearly we can find $t>0$. For hyperbolic $A, t$ is uniquely determined and it is a continuous function of $A$ nowhere equal to zero. Such matrices form an open connected subset of $\operatorname{SL}(2, \mathbb{R})$ (we consider only hyperbolic matrices with trace $>2$ ). So it is enough to check that $t$ is positive for one diagonal matrix, which is obvious.

Definition 2. A finite set $F=\left\{H_{1}, \ldots, H_{n}\right\} \subset \Delta \ell(2, \mathbb{R})$ is called a compatibly hyperbolic (compatibly non-elliptic) family if every product

$$
e^{t_{k} G_{k}} \ldots e^{t_{1} G_{1}} \quad \text { with } G_{i} \in F, t_{i}>0, i=1, \ldots, k
$$

is a hyperbolic (non-elliptic) matrix. Let

$$
\left(\begin{array}{rr}
p & q \\
r & -p
\end{array}\right)=H \in \Delta \ell(2, \mathbb{R}) .
$$

We know that $H$ is hyperbolic if $-\operatorname{det} H=p^{2}+r q>0$, elliptic if $p^{2}+r q<0$, and parabolic if $p^{2}+r q=0$. Hence, geometrically, elliptic matrices form the interior of the cone

$$
S=\{H \in \Delta \ell(2, \mathbb{R}) \mid-\operatorname{det} H \leq 0\} .
$$

For $\left\{H_{1}, \ldots, H_{n}\right\} \subset \diamond \ell(2, \mathbb{R})$ we put

$$
C\left(H_{1}, \ldots, H_{n}\right)=\left\{H \in \delta \ell(2, \mathbb{R}) \mid H=\lambda_{1} H_{1}+\cdots+\lambda_{n} H_{n}, \quad \lambda_{i} \geq 0, \quad i=1, \ldots, n\right\}
$$

i.e. $C\left(H_{1}, \ldots, H_{n}\right)$ is the cone spanned by $H_{1}, \ldots, H_{n}$. Note that $S$ is centrally symmetric, $S=-S$, and $C\left(H_{1}, \ldots, H_{n}\right)$ is not, except for the cases when it is a linear subspace (the whole space, a plane or a line).

THEOREM 3. Let $F=\left\{H_{1}, \ldots, H_{n}\right\} \subset s \ell(2, \mathbb{R})$. F is compatibly hyperbolic (non-elliptic) if and only if

$$
C\left(H_{1}, \ldots, H_{n}\right) \cap S=\{0\}
$$

$\left(C\left(H_{1}, \ldots, H_{n}\right) \cap\right.$ int $\left.S=\varnothing\right)$ and $C\left(H_{1}, \ldots, H_{n}\right)$ is not a proper linear subspace (if $C\left(H_{1}, \ldots, H_{n}\right)$ is a plane then it must be tangent to $\left.S\right)$.

For the proof, we will need the following lemmas:

LEMMA 4. If $F=\left\{H_{1}, \ldots, H_{n}\right\} \subset \triangleleft \ell(2, \mathbb{R})$ is compatibly non-elliptic then

$$
C\left(H_{1}, \ldots, H_{n}\right) \cap \text { int } S=\varnothing .
$$

Proof. We have that $A(t)=e^{i \lambda_{1} H_{1}} e^{i \lambda_{2} H_{2}} \cdots e^{t \lambda_{n} H_{n}}$ for all $t>0$ and fixed $\lambda_{i} \geq 0$ is a non-elliptic matrix in SL $(2, \mathbb{R})$. Hence $d A(t) /\left.d t\right|_{t=0}$ is certainly also non-elliptic i.e. it is outside int $S$. But $d A(t) /\left.d t\right|_{t=0}=\lambda_{1} H_{1}+\cdots+\lambda_{n} H_{n}$.

In the following lemma, we will interpret the conditions from theorem 3 in terms of the configuration of stable and unstable lines of $e^{t_{1} H_{1}}, \ldots, e^{t_{n} H_{n}}, t_{i}>0$. With every

$$
\left(\begin{array}{rr}
p & q \\
r & -p
\end{array}\right)=H \in \delta \ell(2, \mathbb{R})
$$


we associate a quadratic form in $\mathbb{R}^{2}$ invariant for all $e^{t H}, t \in \mathbb{R}$,

$$
Q_{H}(x, y)=-r x^{2}+2 p x y+q y^{2} \text {. }
$$

$H$ is hyperbolic if $Q_{H}$ is indefinite, elliptic if $Q_{H}$ is definite and parabolic if $Q_{H}$ is degenerate.

First, we must point out that the zero lines of $Q_{H}$ are the eigendirections of $H$.

If $H$ is hyperbolic then one, and the same, of the zero lines of $Q_{H}$ is a stable line for all $e^{t H} \in \mathrm{SL}(2, \mathbb{R}), t>0$. To describe which one, consider the space $\mathbb{R} P^{1}$ of all lines in $\mathbb{R}^{2}$ passing through the origin. $e^{t H}$ defines in a natural way a mapping of $\mathbb{R} P^{1}$ into itself which we will also denote by $e^{t H}$. The fixed points of $e^{t H}, t>0$ in $\mathbb{R} P^{1}$ correspond to the stable and unstable lines of the linear mapping, we will denote them by $s$ and $u$ respectively. (They are hyperbolic fixed points $-s$ is unstable and $u$ is stable.)

$Q_{H}$ is not defined on $\mathbb{R} P^{1}$ but the sign of $Q_{H}$ is well defined on $\mathbb{R} P^{1}$. We choose the counterclockwise orientation on $\mathbb{R} P^{1}$. We claim that $Q_{H}$ changes sign from to + on $u$ and from + to - on $s$, with respect to the chosen orientation.

The pattern must be the same for all hyperbolic matrices in $\diamond \ell(2, \mathbb{R})$ since they form a connected set. So it is enough to check it only for one matrix, for instance the diagonal matrix

$$
H=\left(\begin{array}{rr}
1 & 0 \\
0 & -1
\end{array}\right) .
$$

Lemma 5. Let $C\left(H_{1}, H_{2}\right) \cap S=\{0\}$. Then $\lambda_{1} H_{1}+\lambda_{2} H_{2}$ is hyperbolic for all $\lambda_{1}, \lambda_{2}$, $\lambda_{1}^{2}+\lambda_{2}^{2}>0$, if and only if the fixed points of $e^{i H_{1}}, u_{1}, s_{1}$, and of $e^{t H_{2}}, u_{2}, s_{2}$ in $\mathbb{R} P^{1}$ alternate i.e. their order is $u_{1}, u_{2}, s_{1}, s_{2}$ or $u_{1}, s_{2}, s_{1}, u_{2}$ and are all different. $\lambda_{1} H_{1}+\lambda_{2} H_{2}$ is elliptic for some $\lambda_{1}, \lambda_{2}$ if and only if the fixed points of $e^{t H_{1}}$ and $e^{t H_{2}}$ appear in the order $u_{1}, u_{2}, s_{2}, s_{1}$ or $u_{2}, u_{1}, s_{1}, s_{2}$ and are all different.

Proof. If the fixed points alternate and are in particular all different then the quadratic form $\lambda_{1} Q_{H_{1}}+\lambda_{2} Q_{H_{2}}$ has different signs at $u_{1}, s_{1}$ if $\lambda_{2} \neq 0$ and at $u_{2}, s_{2}$ if $\lambda_{1} \neq 0$. Hence the form is indefinite if $\lambda_{1}^{2}+\lambda_{2}^{2}>0$. This is equivalent to the hyperbolicity of $\lambda_{1} H_{1}+\lambda_{2} H_{2}$. If, on the contrary, the fixed points do not alternate, then without loss of generality, we can assume that the order of the fixed points is

$$
u_{1}, u_{2}, s_{2}, s_{1} \text { or } u_{1}, s_{2}, u_{2}, s_{1} \text {, }
$$

where we do not exclude coincidence of some of the points. So when we take invariant lines of $e^{t H_{1}}$ as coordinate axes then

and

$$
Q_{H_{1}}(x, y)=f x y \quad \text { with } f>0,
$$

$$
Q_{H_{2}}(x, y)=a x^{2}+b x y+c y^{2} \quad \text { where } a c \geq 0 \text { and } b(a+c)<0 .
$$

The case $a \geq 0, c \geq 0, b<0$ corresponds to the ordering $u_{1}, s_{2}, u_{2}, s_{1}$ and the case $a \leq 0, c \leq 0, b>0$ to the ordering $u_{1}, u_{2}, s_{2}, s_{1}$. If $a \neq 0$ and $c \neq 0$ then all the fixed points are different.

Clearly, some linear combination $\lambda_{1} Q_{H_{1}}+\lambda_{2} Q_{H_{2}}$ is not indefinite and hence $\lambda_{1} H_{1}+$ $\lambda_{2} \mathrm{H}_{2}$ is not hyperbolic.

If $a>0, c>0, b<0$ then $\lambda_{1} H_{1}+\lambda_{2} H_{2}$ is elliptic for some $\lambda_{1}>0, \lambda_{2}>0$. 
It can also be seen that, with the hypothesis of this lemma, $\lambda_{1} H_{1}+\lambda_{2} H_{2}$ is parabolic only if $\lambda_{1} H_{1}+\lambda_{2} H_{2}=0$. Thus $u_{i}, s_{i}$ are distinct if $C\left(H_{1}, H_{2}\right)$ is not a line.

Proof of theorem 3. Let $u_{1}, \ldots, u_{n}$ and $s_{1}, \ldots, s_{n}$ denote the fixed points of $e^{t H_{1}}, \ldots, e^{t H_{n}}$ in $\mathbb{R} P^{1}$.

Sufficiency. By the condition for all $1 \leq i, j \leq n, C\left(H_{i}, H_{i}\right) \cap S=\{0\}$ and $C\left(H_{i}, H_{j}\right)$ is not a line. Hence, by lemma $5, u_{i} \neq s_{j}$ and $u_{j} \neq s_{i}$. So

$$
\left\{u_{1}, \ldots, u_{n}\right\} \cap\left\{s_{1}, \ldots, s_{n}\right\}=\varnothing
$$

(we do not exclude the possibility that some of the points $u_{1}, \ldots, u_{n}$ or $s_{1}, \ldots, s_{n}$ coincide).

Let us consider a continuous deformation of $H_{1}, \ldots, H_{n}$ in the set of hyperbolic matrices.

$$
H_{i}(t)=t H_{1}+(1-t) H_{i}, \quad 0 \leq t \leq 1 .
$$

We have $C\left(H_{1}(t), \ldots, H_{n}(t)\right) \cap S=\{0\}$ and $C\left(H_{1}(t), \ldots, H_{n}(t)\right)$ is not a line or a plane for all $0 \leq t \leq 1$. Consequently,

$$
\left\{u_{1}(t), \ldots, u_{n}(t)\right\} \cap\left\{s_{1}(t), \ldots, s_{n}(t)\right\}=\varnothing,
$$

where $u_{i}(t), s_{i}(t)$ are the fixed points of $e^{t H_{i}}$ in $\mathbb{R} P^{1}$ corresponding to the unstable and stable lines respectively; $u_{i}(1)=u_{1}, s_{i}(1)=s_{1}, i=1, \ldots, n$. By continuity we conclude that there must be an interval $I \subset \mathbb{R} P^{1}$ such that $u_{i} \in$ int $I$ and $s_{i} \notin I$, $i=1, \ldots, n$. This interval clearly has the property $e^{t H_{i}} I \subset$ int $I, t>0, i=1, \ldots, n$. The same holds for any composition of the matrices $e^{t H_{i}}, t>0, i=1, \ldots, n$. But only hyperbolic matrices in $\operatorname{SL}(2, \mathbb{R})$ have the property that they map some cone (interval in $\mathbb{R} P^{1}$ ) strictly into itself. This ends the proof of sufficiency in the hyperbolic version.

In the non elliptic case, we allow $C\left(H_{1}, \ldots, H_{n}\right)$ to be a line, a plane tangent to $S$ or a cone tangent to $S$. Both a line and a plane tangent to $S$ are subalgebras of $\Delta \ell(2, \mathbb{R})$ corresponding to Lie subgroups of $\operatorname{SL}(2, \mathbb{R})$. The latter is the subgroup of matrices which are triangular in a certain basis, i.e. a subgroup of matrices having a common invariant line. All elements of this subgroup are hyperbolic except for one parameter subgroups of parabolic matrices. So clearly in these cases we have compatible non-ellipticity.

The case of the proper cone tangent to $S$ is similar to the hyperbolic case and we omit the details.

Necessity. In view of lemmas 4 and 5 , we have to prove that $C\left(H_{1}, \ldots, H_{n}\right)$ cannot be a plane (if it is a plane then it must be tangent to $S$ in the non-elliptic case).

Assume on the contrary that for example $C\left(H_{1}, H_{2}, H_{3}\right)$ is a plane. If the plane is tangent to $S$ then $e^{t_{1} H_{1}}, e^{t_{2} H_{2}}$ and $e^{t_{3} H_{3}}$ have a common invariant line which is a stable line for one of them and unstable for another. It follows easily that one of their compositions is parabolic. So assume $C\left(H_{1}, H_{2}, H_{3}\right)$ is not tangent to $S$. For the purpose of performing explicit computations, we will simplify $\mathrm{H}_{1}, \mathrm{H}_{2}, \mathrm{H}_{3}$ by a change of variables. First of all we can make them all symmetric. This is so because by lemma 5 the eigenvectors of $H_{1}$ and $H_{2}$ alternate and so there is a linear 
transformation making both pairs orthogonal simultaneously. $H_{3}$ will become symmetric automatically and we can take care that it is diagonal. Using proposition 1 we can, without loss of generality, assume that for some $t_{1}>0, t_{2}>0$

$$
e^{t_{1} H_{1}}=\left(\begin{array}{cc}
a_{1} & 1 \\
1 & d_{1}
\end{array}\right), \quad e^{t_{2} H_{2}}=\left(\begin{array}{cc}
a_{2} & -1 \\
-1 & d_{2}
\end{array}\right) \quad \text { and } \quad e^{t H_{3}}=\left(\begin{array}{cc}
e^{v t} & 0 \\
0 & e^{-v t}
\end{array}\right)
$$

where $v>0$, while

$$
H_{1}=\left(\begin{array}{cc}
\frac{a_{1}-d_{1}}{2} & 1 \\
1 & \frac{d_{1}-a_{1}}{2}
\end{array}\right), \quad H_{2}=\left(\begin{array}{cc}
\frac{a_{2}-d_{2}}{2} & -1 \\
-1 & \frac{d_{2}-a_{2}}{2}
\end{array}\right) \quad \text { and } H_{3}=\left(\begin{array}{cc}
v & 0 \\
0 & -v
\end{array}\right) \text {. }
$$

By assumption, there are $\lambda_{1}>0, \lambda_{2}>0, \lambda_{3}>0$ such that

$$
\lambda_{1} H_{1}+\lambda_{2} H_{2}+\lambda_{3} H_{3}=0 \text {. }
$$

Hence $a_{1}-d_{1}+a_{2}-d_{2}<0$ i.e. $a_{1}+a_{2}<d_{1}+d_{2}$. But $d_{i}=2 / a_{i}, i=1,2$ since det $e^{t_{i} H_{i}}=1$. We conclude that $a_{1} a_{2}<2$ and $d_{1} d_{2}>2$. We have

$$
\operatorname{tr} e^{t_{1} H_{1}} e^{t H_{3}} e^{t_{2} H_{2}}=e^{v t}\left(a_{1} a_{2}-1\right)+e^{-v t}\left(d_{1} d_{2}-1\right) .
$$

It is straightforward to show that there is a $t>0$ for which the trace above is less than 2 (and positive). Hence $\left\{H_{1}, H_{2}, H_{3}\right\}$ are not even compatibly hyperbolic.

From the proof we derive in addition the following:

Proposition 6. A set $\left\{H_{1}, \ldots, H_{n}\right\} \subset \diamond \ell(2, \mathbb{R})$ is compatibly hyperbolic (non-elliptic) if and only if there is a basis in which all $e^{t H_{i}}, t>0, i=1, \ldots, n$ have positive (non-negative) entries.

Proof. If $\left\{H_{1}, \ldots, H_{n}\right\}$ is compatibly hyperbolic then there is a cone in $\mathbb{R}^{2}$ which is mapped strictly into itself by all $e^{t H_{i}}, t>0, i=1, \ldots, n$. If we choose the sides of the cone as the coordinate axes then all $e^{t H_{i}}, t>0, i=1, \ldots, n$ will become matrices with positive entries.

The sufficiency of the condition is obvious.

We will now express the conditions from theorem 3 in an analytic form. It is enough to do it only for a triple of matrices since $\left\{H_{1}, \ldots, H_{n}\right\}$ is compatibly hyperbolic (non-elliptic) if every triple is. Let

$$
\begin{gathered}
H_{i}=\left(\begin{array}{rr}
p_{i} & q_{i} \\
r_{i} & -p_{i}
\end{array}\right), \\
Q_{H_{i}}(x, y)=-r_{i} x^{2}+2 p_{i} x y+q_{i} y^{2}, \quad i=1,2,3 .
\end{gathered}
$$

Now $C\left(H_{1}, H_{2}, H_{3}\right) \cap S=\{0\}$ and $C\left(H_{1}, H_{2}, H_{3}\right)$ is different from a proper subspace if and only if the quadratic form

$$
\lambda_{1} Q_{H_{1}}+\lambda_{2} Q_{H_{2}}+\lambda_{3} Q_{H_{3}}
$$

is indefinite for all $\lambda_{1} \geq 0, \lambda_{2} \geq 0, \lambda_{3} \geq 0, \lambda_{1}^{2}+\lambda_{2}^{2}+\lambda_{3}^{2}>0$. For the pair of hyperbolic matrices $\left\{H_{i}, H_{j}\right\}$ put $k_{i j}=\operatorname{tr} H_{i} H_{j} / \operatorname{det} H_{i} \operatorname{det} H_{j}$. 
Proposition 7. $\left\{\mathrm{H}_{1}, \mathrm{H}_{2}, \mathrm{H}_{3}\right\}$ is a compatibly hyperbolic family if and only if the quadratic form

$$
\Delta\left(\lambda_{1}, \lambda_{2}, \lambda_{3}\right)=\lambda_{1}^{2}+\lambda_{2}^{2}+\lambda_{3}^{2}+k_{12} \lambda_{1} \lambda_{2}+k_{23} \lambda_{2} \lambda_{3}+k_{13} \lambda_{1} \lambda_{3}
$$

is positive for all $\lambda_{1} \geq 0, \lambda_{2} \geq 0, \lambda_{3} \geq 0, \lambda_{1}^{2}+\lambda_{2}^{2}+\lambda_{3}^{2}>0$.

Proof. Straightforward computation.

The condition from proposition 7 can be expressed explicitly in terms of the coefficients $k_{12}, k_{23}, k_{13}$ but the formulation is so involved that it is of little if any interest. There are clearly simple numerical methods to determine the compatible hyperbolicity of $\left\{\mathrm{H}_{1}, \mathrm{H}_{2}, \mathrm{H}_{3}\right\}$.

For two hyperbolic matrices $\left\{H_{1}, H_{2}\right\}$ the situation is simpler.

PROPOSITION 8. $\left\{\mathrm{H}_{1}, \mathrm{H}_{2}\right\}$ is a compatibly hyperbolic (non-elliptic) pair if and only if $k_{12}>-2\left(k_{12} \geq-2\right)$.

If our family of matrices $F$ contains parabolic matrices then checking compatible non-ellipticity becomes simpler. First, it follows immediately from theorem 3 that a compatibly non-elliptic family of matrices can contain at most two non-colinear (equivalently: non-commuting) parabolic matrices.

Proposition 9. Let $F=\left\{H_{1}, \ldots, H_{n}\right\} \subset s \ell(2, \mathbb{R})$ be a family of hyperbolic matrices and let $P_{1}, P_{2}$ be two parabolic, non-commuting matrices. If $\operatorname{tr} P_{1} P_{2}>0$ and $\operatorname{tr} H_{j} P_{i}>0$, $i=1,2, j=1, \ldots, n$, then $F$ is compatibly hyperbolic.

Proof. For a parabolic matrix $P \in\lrcorner \ell(2, \mathbb{R})$ consider the set $\{X \in \Delta \ell(2, \mathbb{R}) \mid \operatorname{tr} X P>0\}$. Geometrically it is a half-space on one side of the plane tangent to $S$ and containing $P$. It is the half-space which does not contain the part of $S$ in which $P$ lies. In particular tr $P_{1} P_{2}>0$ means that $P_{1}$ and $P_{2}$ lie in different parts of $S$. Hence

$$
K=\left\{X \in \mathcal{O}(2, \mathbb{R})\left\{\operatorname{tr} X P_{1}>0 \text { and } \operatorname{tr} X P_{2}>0\right\}\right.
$$

is a 'quarter' of the space that intersects $S$ along two half-lines passing through $P_{1}$ and $P_{2}$. In particular $K$ is convex and does not contain any plane or line. Hence, by theorem 3 , the conditions are sufficient for hyperbolicity of $F$.

The geometric considerations in the proof above and theorem 3 give us the following criterion:

Proposition 10. $\left\{H_{1}, \ldots, H_{n}\right\} \subset s \ell(2, \mathbb{R})$ is a compatibly hyperbolic family if and only if there are two parabolic matrices $P_{1}, P_{2}$ such that $\operatorname{tr} P_{1} P_{2}>0$ and $\operatorname{tr} H_{j} P_{i}>0$, $j=1, \ldots, n, i=1,2$.

We can formulate one more criterion.

Proposition 11. Let $F=\left\{H_{1}, \ldots, H_{n}\right\} \subset s \ell(2, \mathbb{R})$ be a family of hyperbolic matrices and let $P$ be a parabolic matrix. If $\operatorname{tr} H_{i} P \geq 0, i=1, \ldots, n$, and $k_{i j}>-2,1 \leq i, j \leq n$, then $F$ is compatibly hyperbolic.

The proof is very much in the spirit of previous arguments and we omit it.

One can consider a discrete counterpart of compatible hyperbolicity for a finite set of matrices from $\operatorname{SL}(2, \mathbb{R})$. Theorem 3 provides a sufficient condition for such 
a property but not a necessary condition. Indeed the following theorem holds:

TheOREM 12. Let $F=\left\{H_{1}, \ldots, H_{n}\right\} \subset s \ell(2, \mathbb{R})$ be a family of hyperbolic matrices such that no unstable line of $e^{t H_{i}}, t>0, i=1, \ldots, n$, coincides with any of their stable lines then there is $T>0$ such that $e^{t_{1} G_{1}} \ldots e^{t_{k} G_{k}}, G_{i} \in F, i=1, \ldots, k$, is hyperbolic if $t_{i} \geq T, i=1, \ldots, k$.

Proof. Let $u_{i}, s_{i}$ be the fixed points of $e^{t H_{i}}, t>0$, in $\mathbb{R} P^{1}$ corresponding respectively to the unstable and stable lines. By the assumption there are closed intervals $I_{1}, \ldots, I_{n}, u_{i} \in$ int $I_{i}$, such that $s_{j} \notin \bigcup_{i=1}^{n} I_{i}$. So there is $T>0$ such that if $t \geq T$ then $e^{i H_{j}} I_{j} \subset$ int $I_{j}, 1 \leq i, j \leq n$. It follows that if $G_{1}=H_{i}$ then $e^{t_{1} G_{1}} \cdots e^{t_{k} G_{k}} I_{i} \subset$ int $I_{i}$.

\section{Liapunov exponents}

We fix some norm (for instance the euclidian norm) in $\mathbb{R}^{2}$.

TheOREM 13. Let $F=\left\{H_{1}, \ldots, H_{n}\right\} \subset \Delta \ell(2, \mathbb{R})$. $F$ is compatibly hyperbolic if and only if there are $C>0, d>0$ and a cone $V \subset \mathbb{R}^{2}$ such that

$$
\left\|e^{t_{k} G_{k}} \cdots e^{t_{1} G_{1}} v\right\| \geq C e^{T d}\|v\|
$$

where $G_{i} \in F, t_{i}>0, i=1, \ldots, k, T=t_{1}+\cdots+t_{k}$ and $v \in V$.

Proof. Sufficiency is obvious. For the proof of necessity we can, by proposition 6, without loss of generality, assume that all $e^{t H_{i}}, t>0, i=1, \ldots, n$ have positive entries. Hence, by proposition 1 ,

$$
H_{i}=\left(\begin{array}{rr}
p_{i} & q_{i} \\
r_{i} & -p_{i}
\end{array}\right)
$$

with $q_{i}>0, r_{i}>0, i=1, \ldots, n$. Let $q=\min _{i} q_{i}, r=\min _{i} r_{i}, p=\max _{i}\left|p_{i}\right|$. Consider a linear system of differential equations

$$
\dot{u}=M(t) u, \quad u \in \mathbb{R}^{2},
$$

where $M(t)$ is piecewise constant

$$
M(t)=G_{i} \quad \text { if } t_{1}+\cdots+t_{i-1}<t \leq t_{1}+\cdots+t_{i-1}+t_{i}, \quad i=1, \ldots, n .
$$

The vector on the left hand side of the inequality is equal to $u(T)$ where $u(t)$, $0 \leq t \leq T$ is a solution of (1) with the initial value $u(0)=v$.

We will find a Liapunov function for (1) in the positive octant (this is the cone $v)$. Consider a quadratic function

$$
f(x, y)=a x^{2}+b y^{2}+2 x y .
$$

There are choices of $a>0, b>0$ and $g>0$ such that

$$
\frac{d f}{d t}(x, y) \geq g f(x, y) \quad \text { for } x \geq 0, y \geq 0 .
$$

Indeed we have for $x \geq 0, y \geq 0$,

$$
\frac{d f}{d t}(x, y) \geq 2\left((r-a p) x^{2}+(q-b p) y^{2}+(a q+b r) x y\right) \geq g\left(a x^{2}+b y^{2}+2 x y\right),
$$

if $a, b, g$ are sufficiently close to zero. As a consequence, we have

$$
f(x(t), y(t)) \geq e^{t g} f(x(0), y(0)), \quad 0 \geq t \geq T,
$$


if $x(0) \geq 0, y(0) \geq 0$. On the other hand there is a constant $\gamma>0$ such that for $v=(x, y), x \geq 0, y \geq 0$

$$
\gamma^{-1}\|v\| \leq \sqrt{f(x, y)} \leq \gamma\|v\| .
$$

Combining the estimates above we get the desired inequality.

By similar arguments we can obtain:

THEOREM 14. If $F=\left\{H_{1}, \ldots, H_{n}\right\} \subset \Delta \ell(2, \mathbb{R})$ is compatibly hyperbolic and $F \cup$ $\left\{P_{1}, P_{2}\right\}$ is compatibly non-elliptic where $P_{1}, P_{2}$ are parabolic matrices then there are constants $C>0, d>0$ and a cone $V \subset \mathbb{R}^{2}$ ( $V$ is bounded by invariant lines of $P_{1}, P_{2}$ if they are different) such that

$$
\left\|e^{t_{k} G_{k}} \cdots e^{t_{1} G_{1}} v\right\| \geq C e^{T d}\|v\|,
$$

where $G_{i} \in F \cup\left\{P_{1}, P_{2}\right\}, t_{i}>0, i=1, \ldots, k, T=\sum_{G_{i} \in F} t_{i}$ and $v \in V$.

We are now ready to formulate our criterion for positivity of the maximal Liapunov characteristic exponent. Let $T: X \rightarrow X$ be a measure preserving transformation of the probability space $(X, \mu)$ and let $A: X \rightarrow \operatorname{SL}(2, \mathbb{R})$ be a measurable mapping. We assume that the values of $\boldsymbol{A}$ are non-elliptic matrices. Without loss of generality we can assume that traces of matrices in $A(X)$ are $\geq 2$ (multiplication of some of the matrices by -1 does not affect Liapunov exponents).

Case I (one parabolic matrix). Suppose that $A(X)=\left\{A_{1}, \ldots, A_{n}, B_{1}\right\}$ where $A_{i}$ are hyperbolic, $i=1, \ldots, n$, and $B_{1}$ is parabolic (i.e. $\operatorname{tr} B_{1}=2$ ).

If, for every $1 \leq i, j \leq n$

$$
2 \operatorname{tr} A_{i} A_{j}-\operatorname{tr} A_{i} \operatorname{tr} A_{j}>-\sqrt{\left(\operatorname{tr} A_{i}\right)^{2}-4} \sqrt{\left(\operatorname{tr} A_{j}\right)^{2}-4}
$$

and for every $1 \leq i \leq n$

$$
\operatorname{tr}\left(B_{1}-I\right) A_{i} \geq 0 \text { and }\left(\bigcap_{n=0}^{\infty} T^{-n}\left(A^{-1}\left\{B_{1}\right\}\right)\right)=0
$$

then the m.L.c.e. is positive almost everywhere.

Case II (two parabolic matrices). Suppose that $A(X)=\left\{A_{1}, \ldots, A_{n}, B_{1}, B_{2}\right\}$ where $A_{i}$ are hyperbolic, $i=1, \ldots, n$, and $B_{1}, B_{2}$ are parabolic (i.e. $\operatorname{tr} B_{1}=\operatorname{tr} B_{2}=2$ ). If $\operatorname{tr} B_{1} B_{2}>2$ and for every $1 \leq i \leq n, j=1,2$,

$$
\operatorname{tr}\left(B_{j}-I\right) A_{i}>0 \text { and }\left(\bigcap_{n=0}^{\infty} T^{-n}\left(A^{-1}\left\{B_{1}, B_{2}\right\}\right)\right)=0 \text {, }
$$

then the m.L.c.e. is positive almost everywhere.

Proof. We have $A_{i}=e^{t H_{i}}, t>0$, with hyperbolic $H_{i} \in \diamond \ell(2, \mathbb{R}), i=1, \ldots, n$, and $B_{j}=e^{t P_{i}}, t>0$, with parabolic $P_{j} \in \delta \ell(2, \mathbb{R}), j=1,2$.

Using proposition 1, we express sufficient conditions for compatible hyperbolicity of $\left\{H_{1}, \ldots, H_{n}\right\}$ using proposition 9 and proposition 11 and we get the conditions above. Now we are in a position to use theorem 14 which gives us immediately positivity of the m.L.c.e. 


\section{Application}

In this final section, we will deal with a particular kind of measure-preserving transformation on the 2-dimensional torus $\mathbb{J}^{2}$,

$$
T\left(x_{1}, x_{2}\right)=\left(x_{2},-x_{1}+C x_{2}+f\left(x_{2}\right)\right)
$$

where $f$ is a periodic function and $C$ is an integer constant. We will use the square $\left[-\frac{1}{2}, \frac{1}{2}\right] \times\left[-\frac{1}{2}, \frac{1}{2}\right]$ in $\mathbb{R}^{2}$ as the fundamental domain of $\mathbb{T}^{2}$. Let $S\left(x_{1}, x_{2}\right)=\left(x_{2}, x_{1}\right) . T$ is $S$-reversible i.e. $S \circ T \circ S=T^{-1}$.

When $|C|$ is $>2$ and $f$ is a smooth, $C^{1}$-small function, we have an Anosov diffeomorphism. It is also of interest that for $|C| \leq 2$ and an appropriate 'perturbation' $f$, the m.L.c.e. are positive in some part of $\mathbb{J}^{2}$, thus ensuring strong mixing properties of $T$ (cf. [3], [4]). The case $C=2$ corresponds to perturbations of the twist map and was treated in [4] and [5]. For $C=-2,-1,1$, one can get results similar to those of [4] by essentially the same approach (this was done explicitly in [1]). We will obtain interesting dynamical behaviour for the case $C=0$ using the criterion developed in previous sections (since it was proved in [1] that the criterion of [4] doesn't work in this case).

Thus, we study $T\left(x_{1}, x_{2}\right)=\left(x_{2},-x_{1}+f\left(x_{2}\right)\right)$ with

$$
f(t)=\left\{\begin{aligned}
-a t-d & \text { on }\left[-\frac{1}{2}, 0\right], \\
a t+d & \text { on }\left[0, \frac{1}{2}\right] .
\end{aligned}\right.
$$

So $T$ is linear in $B_{+}$and $B_{-}$where

$$
B_{ \pm}=\left\{(x, y) \mid 0 \leq \pm y \leq \frac{1}{2},-\frac{1}{2} \leq x \leq \frac{1}{2}\right\} .
$$

The matrix of $T$ (or $D T$ ) in $B_{+}$is $D_{1}$ and in $B_{-}$is $D_{2}$ where:

$$
D_{1}=\left(\begin{array}{rr}
0 & 1 \\
-1 & a
\end{array}\right), \quad D_{2}=\left(\begin{array}{rr}
0 & 1 \\
-1 & -a
\end{array}\right)
$$

We specify $d$ to be such that $\left(-\frac{1}{4},-\frac{1}{4}\right)$ and $\left(\frac{1}{4}, \frac{1}{4}\right)$ are fixed points for $T$ i.e. $d=\frac{1}{4} a+\frac{1}{2}$.

For $|a|<2$, our transformation is a rotation about the fixed point $\left(-\frac{1}{4},-\frac{1}{4}\right)$ in $B_{-}$ and about the fixed point $\left(\frac{1}{4}, \frac{1}{4}\right)$ in $B_{+}$. So we have two invariant 'elliptic islands' $I_{ \pm}=\bigcap_{k=-\infty}^{\infty} T^{k}\left(B_{ \pm}\right)$. These domains are ellipses if the rotation is irrational and polygons if it is rational. We shall prove the following:

TheOREM 15. For $a=2 \cos \pi / n, n=2,3, \ldots, T$ has positive m.L.c.e. almost everywhere in $\mathbb{T}^{2} \backslash\left(I_{+} \cup I_{-}\right)$. For the values of a described in this theorem, $I_{+}$and $I_{-}$ are polygons with $2 n$ sides symmetric with respect to the diagonal (i.e. $S\left(I_{ \pm}\right)=I_{ \pm}$) in view of $S$-reversibility. Outside of them the interaction of different rotations produces strong mixing properties.

Proof. We will consider the return map $\tilde{T}: B_{+} \cap C_{-} \varsigma$ where $C_{ \pm}=T\left(B_{ \pm}\right)=$ $\left\{\left(x_{1}, x_{2}\right) \mid 0 \leq \pm x_{1} \leq \frac{1}{2}\right\}$. Clearly it is enough to prove that $\tilde{T}$ has positive m.L.c.e.

$D \tilde{T}$ is equal to $D_{2}^{j} D_{1}^{i}$ where $i$ is the number of times a point from $B_{+} \cap C_{-}$stays in $B_{+}$before leaving it and $j$ is the number of times it stays in $B_{-}$before returning to $B_{+}$. The crucial observation is that $1 \leq i, j \leq n-1$ and $2 \leq i+j \leq n$. The latter is 


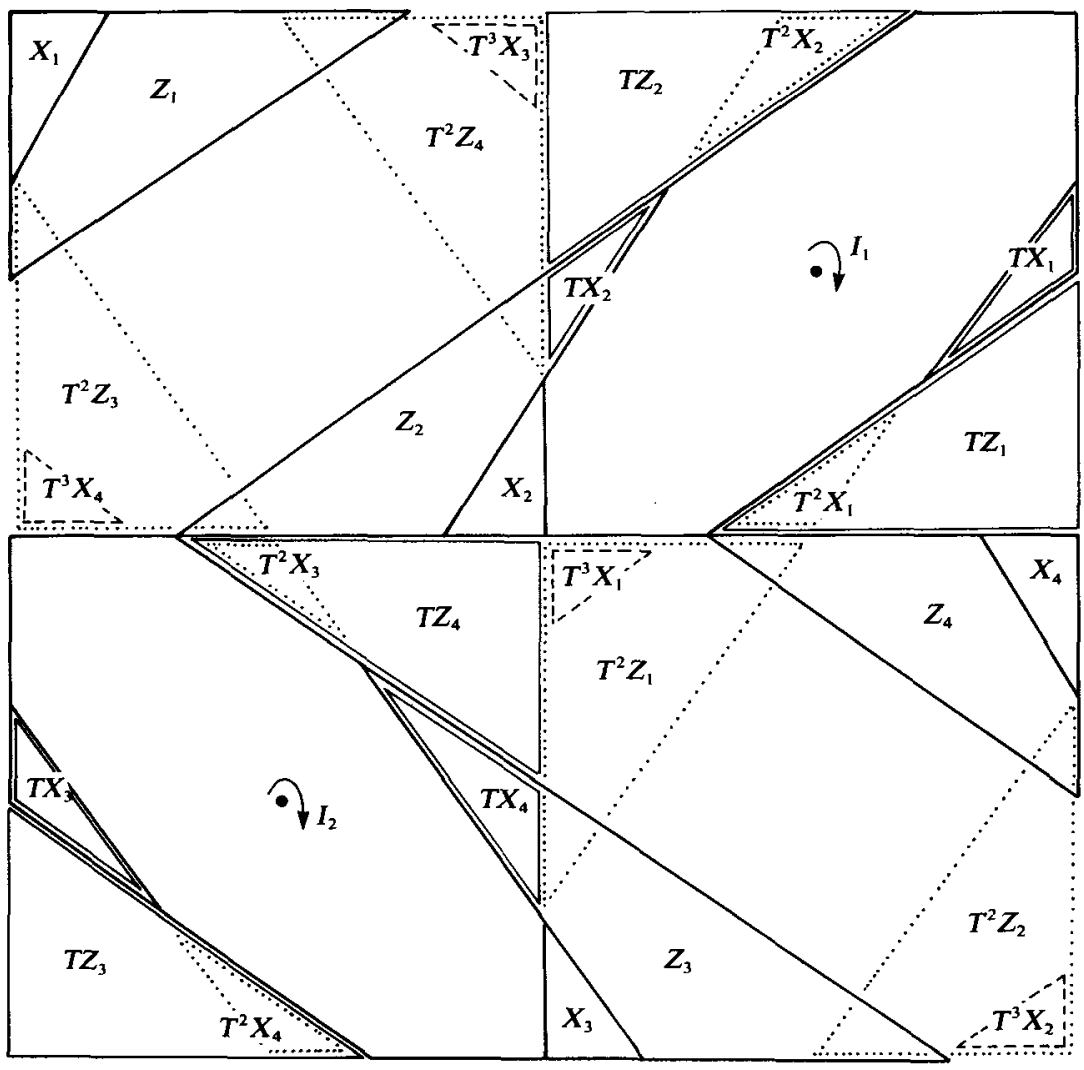

FIGURE 3-1. Case $n=4$. The domains $I_{i}$ are the periodic elliptic islands, the zones $Z_{i}$ stay only twice (i.e. $Z_{i}$ and $T\left(Z_{i}\right)$ ) in the same part of the torus (i.e. where it's always the same differential) $B_{+}$or $B_{-}$ before going to the other half of $\nabla^{2}$. The $X_{i}$ stay for 3 iterations $\left(X_{i}, T\left(X_{i}\right)\right.$ and $\left.T^{2}\left(X_{i}\right)\right)$ in the same half-part of the torus. So the only allowed products are $D_{2} D_{1}, D_{2} D_{1}^{2}, D_{2} D_{1}^{3}, D_{2}^{2} D_{1}, D_{2}^{2} D_{1}^{2}$ and $D_{2}^{3} D_{1}$.

messy to prove but can be seen fairly easily geometrically as for example in the case $n=4$ shown in figure 3-1.

$D_{1}^{i}$ and $D_{2}^{j}$ can be expressed in the following form (as proved in [5])

$$
\begin{aligned}
& D_{1}^{i}=\frac{(-1)^{i+1}}{\sin \pi / n}\left(\begin{array}{cc}
\sin (i-1) \pi / n & \sin i \pi / n \\
-\sin i \pi / n & -\sin (i+1) \pi / n
\end{array}\right) \\
& D_{2}^{j}=\frac{1}{\sin \pi / n}\left(\begin{array}{cc}
-\sin (j-1) \pi / n & \sin j \pi / n \\
-\sin j \pi / n & \sin (j+1) \pi / n
\end{array}\right)
\end{aligned}
$$

and thus

where

$$
D_{1}^{i} D_{2}^{j}=\frac{(-1)^{i+1}}{\sin ^{2} \pi / n}\left(\begin{array}{ll}
d_{11} & d_{12} \\
d_{21} & d_{22}
\end{array}\right)
$$

$$
\begin{aligned}
& d_{11}=-\sin (j-1) \pi / n \sin (i-1) \pi / n-\sin i \pi / n \sin j \pi / n \\
& d_{12}=-\sin (j-1) \pi / n \sin i \pi / n-\sin j \pi / n \sin (i+1) \pi / n
\end{aligned}
$$




$$
\begin{aligned}
& d_{21}=-\sin j \pi / n \sin (i-1) \pi / n-\sin i \pi / n \sin (j+1) \pi / n \\
& d_{22}=-\sin j \pi / n \sin i \pi / n-\sin (j+1) \pi / n \sin (i+1) \pi / n .
\end{aligned}
$$

So we have

$$
\begin{aligned}
\operatorname{tr} D_{1}^{i} D_{2}^{j}= & \frac{(-1)^{i}}{\sin ^{2} \pi / n}[\sin (j-1) \pi / n \sin (i-1) \pi / n+2 \sin i \pi / n \sin j \pi / n \\
& +\sin (j+1) \pi / n \sin (i+1) \pi / n] \\
= & \frac{(-1)^{i}}{\sin ^{2} \pi / n}\left[(\sin i \pi / n \sin j \pi / n)\left(2+2 \cos ^{2} \pi / n\right)\right. \\
& \left.+2 \cos j \pi / n \cos i \pi / n \sin ^{2} \pi / n\right] .
\end{aligned}
$$

After multiplying the matrices $D_{2}^{j} D_{1}^{i}$ by -1 when necessary, we see that all the traces are $\geq 2$. The trace is equal to 2 for $i=n-1, j=1$ and $i=1, j=n-1$.

We put

$$
B_{1}=\left(\begin{array}{cc}
1 & 4 \cos \pi / n \\
0 & 1
\end{array}\right), \quad B_{2}=\left(\begin{array}{cc}
1 & 0 \\
4 \cos \pi / n & 1
\end{array}\right),
$$

and let $\left\{A_{1}, \ldots, A_{n}\right\}$ be the set of all hyperbolic matrices $D_{2}^{j} D_{1}^{i}$ multiplied by -1 if necessary.

Now we apply the criterion from $\S 2$ to $\tilde{T}: B_{+} \cap C_{-} \circlearrowleft$ and $A: B_{+} \cap C_{-} \rightarrow \operatorname{SL}(2, \mathbb{R})$, $A(x)=D \tilde{T}_{x}$ (normalised to give trace $\geq 2$ ). So we have to check that

$$
\begin{gathered}
\operatorname{tr} B_{1} B_{2}>2, \\
\operatorname{tr}\left(B_{k}-I\right) A_{i}>0,
\end{gathered}
$$

and

$$
\left(\bigcap_{n=0}^{\infty} \tilde{T}^{-n}\left(A^{-1}\left\{ \pm B_{1}, \pm B_{2}\right\}\right)\right)=0
$$

Since

$$
\begin{aligned}
B_{1} B_{2} & =\left(\begin{array}{cc}
1 & 4 \cos \pi / n \\
0 & 1
\end{array}\right)\left(\begin{array}{cc}
1 & 0 \\
4 \cos \pi / n & 1
\end{array}\right) \\
& =\left(\begin{array}{cc}
1+16 \cos ^{2} \pi / n & 4 \cos \pi / n \\
4 \cos \pi / n & 1
\end{array}\right),
\end{aligned}
$$

it's obvious that (2) is satisfied.

(3) means in our case that the matrices $\boldsymbol{A}_{i}$ have all positives entries. The property (4) is easily obtained from geometric considerations. Thus the m.L.c.e. are positive almost everywhere in $\mathbb{T}^{2} \backslash\left(I_{+} \cup I_{-}\right)$.

Acknowledgments. We thank the Fonds National de la Recherche Scientifique of Belgium which made possible the stay of Maciej Wojtkowski at F.N.D.P. during which this work was begun, the Facultes Notre Dame de la Paix at Namur which supported the research of Eric Cornelis for this paper and finally the University of California, Berkeley, where Maciej Wojtkowski wrote part of this paper on leave from Warsaw University, Poland. We also want to thank the referees for their helpful remarks. 
E. Cornelis is a Research Assistant of the National Fund for Scientific Research (Belgium).

\section{REFERENCES}

[1] E. Cornelis. Sur les propriétés ergodiques de quelques transformations linéaires par morceaux du tore. Master's thesis, F.N.D.P. Namur, 1982.

[2] V. I. Oseledec. A multiplicative ergodic theorem. Lyapunov characteristic numbers for dynamical systems. Trans. Moscow Math. Soc. 19 (1968), 197-231.

[3] Ya. B. Pesin. Characteristic Lyapunov exponents and smooth ergodic theory. Russian Math. Surveys 32-4 (1977), 55-114.

[4] M. Wojtkowski. A model problem with the coexistence of stochastic and integrable behaviour. Commun. Math. Phys. 80 (1981), 453-464.

[5] M. Wojtkowski. On the ergodic properties of piecewise linear perturbations of the twist map. Ergod. Th. \& Dynam. Sys. 2 (1982), 525-542. 